\title{
Développement et transfert international des technologies avancées dans le domaine de la télématique pour l'environnement - l'expérience du LHF
}

\author{
Development and international transfer of advanced technologies in the \\ domain of telematics for environment - LHF's experience
}

par J. Cunge, M. Erlich et J-L. Rahuel

Laboratoire d'Hydraulique de France - LHFI

Since its founding in 1987 the LHF activity in the domain of water resources (continuation of SOGREAH's R\&D tradition in the field), consisted in development and implementation of applications of Information and Communication technologies (ICT) integrated with hydraulic and hydrological science. LHF has played an important role, among other European companies and institutes, in developing a new technology called Hydroinformatics, which is not in fact an application of ICT to water resources, but synergetic integration of both. This innovative activity allowed $L H F$, on one hand, to influence up to some extend the evolution of thought in water domain in France and, on the other hand, to propose ideas and solutions of French school abroad. In order to illustrate this experience the following four aspects of LHF's activity during last 10 years are presented in the paper :

- participation in the definition and execution of the 4th Framework RDT of European Union,

- development and implementation of the operational hydroinformatics systems (flood risk management in Shangai) or advanced prototypes (flash floods forecasting in France),

- developiment of new technologies and hydroinformatics systems having operational future (such as Integrated System and Services for the Management of Agricultural Pollution - EUREKA/ISMAP or methodology for interpretation of simulation results in imprecise environment using fuzzy logic approach),

- development of new 2-dimensional approach to river engineering studies.

Examples and study cases of respective applications are given in the paper.

\section{I — TÉLÉMATIQUE ET ENVIRONNEMENT : DÉFINITION ET SUIVI DES PROGRAMMES EUROPÉENS}

Au niveau européen la recherche dans le domaine des ressources en eau était, depuis le commencement des programmes de la Commission Européenne (CE), financée par le programme de la DGXII " Climat et Environnement ". Ce programme s'adressait essentiellement aux scientifiques et ne visait pas des applications immédiates. Les résultats des projets permettaient certes d'élargir la base scientifique des connaissances mais restaient éloignés du champ d'intérêt de l’ingénieur.

En parallèle une autre doctrine, fondée sur le succès et la vitesse foudroyante de développement des Technologies d'Informations et des Communications (TIC) se mettait en place au niveau de l'Union, à la DGXIII. En 1993 la CE a décidé de créer dans le cadre du 4e PCRD, à l'intérieur du très vaste programme "Applications Télématiques", un Secteur " Télématique et Environnement ". Le LHF a participé en tant que Chef de file, puis Rapporteur, à la définition de la thématique de ce Secteur, l'appel d'offres correspondant pour les projets de la recherche étant lancé en décembre 1994. L'idée de base proposée par le LHF était que la synergie entre les thèmes Télématique et Environnement soit mise en œuvre à travers des approches et des systèmes de gestion environnementale en liant trois domaines physiques : eau-airsol. Chaque projet doit élaborer un " démonstrateur " et aboutir à sa validation sur le(s) site(s) d'implantation. Suite au premier et au deuxième (1997) appel d'offres, 26 projets ont été retenus, la Commission fournissant près de $30 \mathrm{MECU}$ de subventions. L'objectif est de démontrer l'utilité des solutions télématiques pour la protection et l'amélioration de l'environnement en développant une série d'outils puissants

1 Le LHF, une Société Anonyme, a été absorbé par SOGREAH courant 1998 
permettant aux citoyens, à l'industrie et aux administrations de partager l'information, de collecter rapidement des données, de prendre des décisions bien fondées. Des 26 projets, 12 traitent explicitement des problèmes de ressources en eau tels que : gestion de crises (crues), gestion de la pollution et de la qualité de l'eau, gestion des données distribuées et de leur transformation en informations destinées aux tableaux de bord pour les décideurs et aux observatoires pour le public. 12 projets de la première "vague " se terminent à l'automne 1998, les autres en 2000, mais déjà nous savons que le Secteur "Télématique et Environnement" du 4e PCRD est un succès et ses conclusions servent actuellement à l'élaboration de la thématique du 5e Programme Cadre. Grâce à une vision dynamique, en partant de la problématique de l'Eau, en la globalisant vers des problèmes de l'environnement durable et en cherchant la synergie avec les TIC, il a été donné à une petite société d'influencer la politique européenne de financement des études et de la recherche d'un secteur dans le domaine de l'eau pendant deux Programmes Cadres. Le LHF participe d'ailleurs à l'un des projets à savoir le projet horizontal ANIMATE d'appui aux autres projets, en y apportant son expertise pour aider les projets dans la tâche de validation de leurs démonstrateurs [1].

\section{II — SYSTÈMES HYDROINFORMATIQUES OPÉRATIONNELS}

\subsection{Système d'aide à la gestion en temps réel des risques d'inondation de Shanghaï}

Ce projet, et quelques autres, ont été mis en œuvre par la CE/DGXIII en coopération avec " La Commission d'Etat des Sciences et Technologies " au titre de réalisations pilotes afin de faire pénétrer les technologies de l'information et de la communication, et plus particulièrement les standards européens, dans ce vaste pays à l'économie émergente. L'application de ces technologies d'innovation au domaine de l'environnement, et dans le cas présent celui de la gestion de l'eau, constitue la spécificité du projet dont la Maîtrise d'œuvre a été confiée au LHF. Ce projet est un exemple remarquable de transfert de connaissances et de technologie, de l'Union Européenne et en particulier de la France, vers un pays en voie de développement rapide, qui souhaite gommer au plus vite son retard technologique inhérent à son récent isolement politique : la République Populaire de Chine.

Shanghaï (13 millions d'habitants) est le premier centre économique et le premier port de la Chine après Hong-Kong. La situation de Shanghaï sur les bords de la rivière Huang-Pu, affluent deltaïque du Yangtze, est à la fois exceptionnelle et périlleuse. La rivière Huang-Pu a en effet permis d'aménager un port maritime qui peut accueillir les bateaux océaniques et les terres du delta du Yangtze qui entourent Shanghaï sont également très fertiles. En revanche, Shanghaï vit sous la menace permanente du risque d'inondations. Celles-ci peuvent aussi bien provenir de la terre (" amont") sous l'effet de fortes précipitations de mousson sur le bassin (422 $\mathrm{mm}$ en un jour en $1977,140 \mathrm{~mm}$ en une heure en 1985) que de la mer ("aval ") lors de la conjugaison d'une marée de vive eau et de la surélévation du niveau de la mer générée par la dépression atmosphérique et les vents violents associés au passage d'un typhon (le niveau de la mer peut alors atteindre $5 \mathrm{~m}$ à comparer au niveau du sol à Shanghaï qui se situe partout entre $2 \mathrm{~m}$ et $4 \mathrm{~m}$ ). Les causes " amont" et " aval " de l'inondation peuvent en outre se combiner pour générer des situations catastrophiques.
Des moyens structurants ont été mis progressivement en place par les ingénieurs chinois pour protéger la ville : digues de plus en plus hautes, portes à marées, barrage antitempête, déviation de cours d'eau, stations de pompage en zone urbaine. Ces moyens assurent aujourd'hui un bon niveau de protection de la ville, néanmoins le risque demeure. En plus d'une éducation de la population à la culture du risque et de sa préparation à l'éventualité d'une inondation, auxquelles veille la Municipalité, le dispositif se devait d'être complété par un système performant de gestion et de prévision opérationnelle du risque d'inondation. Le système préexistant, basé sur une technologie dépassée et peu fiable, reflétant en outre la difficulté de faire coopérer les diverses administrations de la ville, était parfaitement inadapté pour gérer la crise.

Le système mis en place dans le cadre du projet UE-Chine (1991-1996) et opérationnel depuis juin 1996 visait à remédier aux carences du dispositif actuel en valorisant au mieux la technologie et le savoir-faire français et européen en hydrologie, en hydraulique et dans le domaine des technologies de l'information et de la communication.

L'architecture du système est présentée sur la figure 1. Il comprend plusieurs niveaux verticalement intégrés. II est en outre configuré pour pouvoir couvrir, à terme, la totalité de la zone urbaine de Shanghaï par un réseau de stations d'acquisition :

- Un réseau de télémétrie constitué de plusieurs grappes télémétriques équipées de capteurs reliés à des stations d'acquisition déportées qui mesurent et transmettent en temps réel les paramètres hydro-météorologiques sensibles : précipitations, niveau d'eau, vitesse et direction du vent. Chaque grappe est équipée d'un sous-centre télémétrique comprenant un frontal radio et un concentrateur de données. Les stations sont reliées au sous-centre télémétrique par des liaisons radio UHF ou VHF.

- Un réseau dédié de télécommunications point-multipoints par faisceaux hertziens (fréquence $1,5 \mathrm{GHz}$ ) d'une capacité de $2 \mathrm{Mbit} / \mathrm{s}$ reliant entre elles 13 stations abonnées. Ces stations situées dans les sous-centres de l'Administration de l'Eau, dans le Centre Météorologique de Shanghaï et dans le Centre de Gestion du bassin du lac Tai Hu, permettent de connecter des téléphones, télécopies, télex, et des terminaux pour la transmission des données à la vitesse maximale de 9600 bits/s. Ce réseau, qui utilise la technologie AMRT (Accès Multiple par Répartition dans le Temps), est la colonne vertébrale du système. Le centre du réseau coïncide avec le Centre de Prévision. Il comprend notamment un $\mathrm{PABX}$ qui gère les appels téléphoniques et à travers lequel le réseau dédié peut être relié au réseau public Chinois.

- Des terminaux X et des micro-ordinateurs de type PC. Ce mini-ordinateur supporte le logiciel nécessaire au fonctionnement du système : communication, bases de données. Le Centre de Prévision est équipé d'un mini-ordinateur HP9000-817s, serveur d'un réseau hydrologique, synoptiques des alertes, atelier de simulation de la prévision, prévision en temps réel. Le logiciel encapsule notamment la plate-forme de prévision MISTERE du LHF et des modèles de prévision hydrologiques et hydrauliques de la bibliothèque de méthodes de MISTERE et des modèles chinois de prévision de trajectoires de typhons et de marées-tempêtes.

On peut trouver plus des détails concernant ce projet dans les références $[2,3]$. 


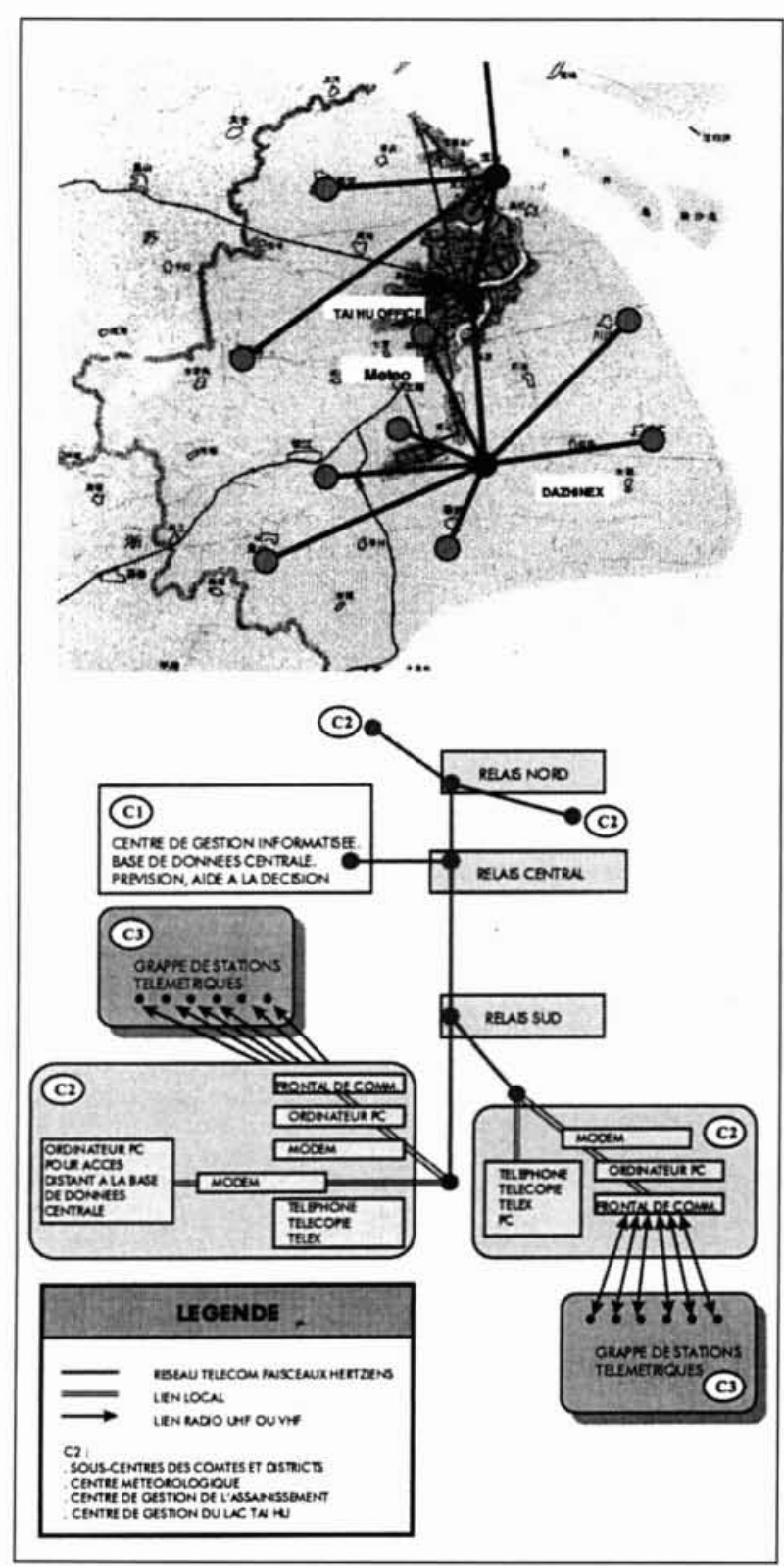

1. Architecture du système de télécommunication, transmission des données et de prévision installé à Shanghaï.

\subsection{Projet des crues éclairs en France}

En s'appuyant sur les progrès scientifiques et technologiques, la Direction de l'Eau du ministère de l'Environnement et de l'Aménagement du Territoire mène depuis plusieurs années des actions visant à maintenir et à améliorer l'efficacité des systèmes d'annonce de crues. Grâce à ces actions on a assisté notamment au progrès de la standardisation des réseaux de télémesures en temps réel et l'installation de tels réseaux dans la majorité des bassins génériques de crues des cours d'eau importants. Parallèlement, les premières installations intégrées permettant d'effectuer en temps réel la prévision à partir d'un réseau d'acquisition de données voyaient le jour dans quelques services d'annonce de crues (SAC) français.

Les efforts se sont d'abord portés sur l'équipement des grands cours d'eau générateurs de " crues de plaines ". Pour ces crues relativement lentes, les réseaux télémétriques de pluviomètres et de limnimètres, d'une densité raisonnable,

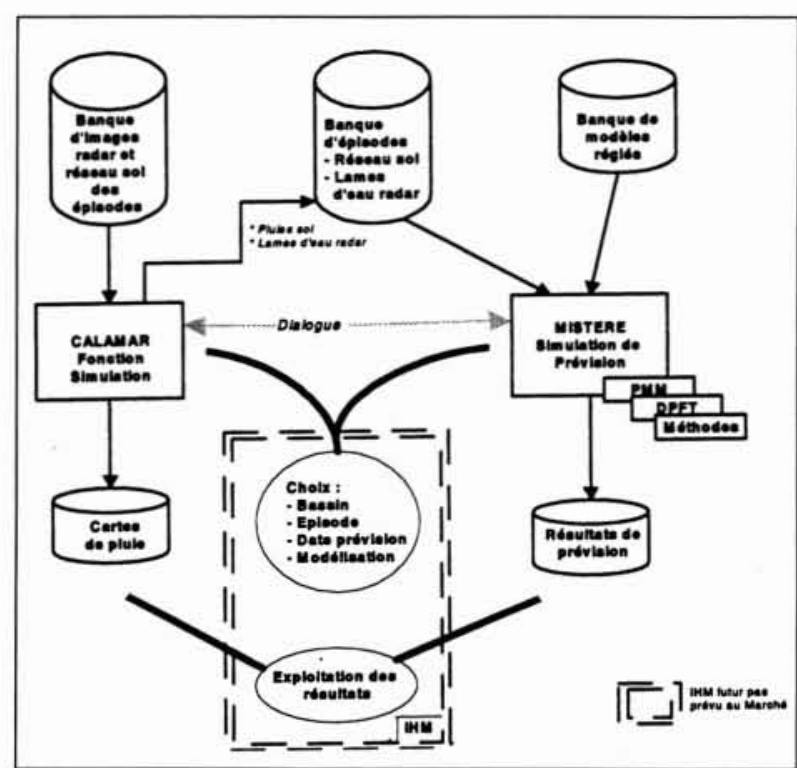

2. Principes architecturaux du prototype de l'outil de prévision de crues-éclairs.

permettent d'acquérir en temps réel les données nécessaires pour décrire correctement leur genèse ainsi que la propagation de la crue le long des cours d'eau.

La spécificité des bassins en zone urbaine mise à part, il existe en fait deux types de crues et deux types de besoins dans le domaine de la prévision : les " crues de plaines " précitées et les " crues-éclairs ". Les différences essentielles, du point de vue de la gestion des risques, concernent l'appréhension des phénomènes et le temps de leurs durées.

En ce qui concerne les " crues-éclairs ", les phénomènes générateurs de catastrophes correspondent à des durées de quelques heures. Ce sont en général des pluies fortement convectives, orages ou successions d'orages, spatialement très hétérogènes et sur des superficies très faibles (Nîmes 1988, Privas 1990, Avignon et Orange 1991, Vaison-LaRomaine et Ardèche 1992, Marseille 1993, etc.). La densité habituelle des pluviomètres installés sur ces bassins versants est souvent insuffisante pour percevoir et décrire ces phénomènes pluvieux à conséquences catastrophiques. La difficulté d'appréhender les phénomènes à l'aide de réseaux classiques de mesure (pluviomètres et limnimètres) rend évidemment encore plus difficile la création de systèmes de prévision fondés sur l'ensemble mesures/modélisations. De plus, l'expertise du prévisionniste est entièrement conditionnée par les délais très courts d'évolution du phénomène, et l'apport d'outils de synthèse et d'aide à la décision est donc nécessaire.

Afin de pouvoir mieux appréhender la mesure des précipitations, le ministère de l'Environnement et de l'Aménagement du Territoire a décidé, il y a quelques années, dans le cadre d'une convention passée avec Météo France, de financer des radars complémentaires au réseau ARAMIS et d'équiper les SAC en outil Météotel. Il a également soutenu des projets pilotes d'exploitation quantitative des images radar de pluies.

Dans ce contexte, le ministère a souhaité prolonger les actions pilotes déjà engagées par un projet fédérateur des compétences au niveau français dans le domaine de la prévision. Il a ainsi confié au groupement composé de LHF (leader du projet), EDF-DTG et RHEA [4], un marché d'un 
montant dépassant $5 \mathrm{MF}$ et ayant pour objet la construction et la validation du prototype d'un outil intégré “ MesuresModélisation-Radar ". Cet outil devrait permettre, sur les bassins versants de moins de $2000 \mathrm{~km}^{2}$, de quantifier la pluie et d'en modéliser ses conséquences hydrauliques avec une précision et un délai permettant la prévision des crues-éclairs.

Si la validation de l'outil est concluante, une étape ultérieure au projet pourrait être d'assurer l'implantation opérationnelle sur un ou deux sites pilotes du prototype d'un produit intégré. Une fois le prototype du produit éprouvé, il pourrait faire l'objet d'un développement industriel et être commercialisé sur le marché national et international. Notons qu'une fois le principe du prototype validé sur les sites-tests, il sera possible d'intégrer dans cet outil, grâce à l'architecture générique de la plate-forme MISTERE, n'importe quel modèle usuel hydraulique (propagation des ondes de crues) ou hydrologique (transformation pluie/débit).

Le projet a démarré en août 1996. Deux sites-tests ont été retenus : le Gardon d'Anduze dans le Gard et le Lignon-duVelay en Haute-Loire. Les données sol (limnimètres et pluviomètres) et les images radar ont été collectées et traitées. Une modélisation a été mise en œuvre sur chacun des sites, basée notamment sur l'utilisation du modèle DPFT de transformation pluie-débit de EDF-DTG [5]. L'Outil de prévision qui intègre la partie d'exploitation de la plate-forme de prévision MISTERE [6] du LHF, le système CALAMAR [7] d'élaboration de lames d'eau radar de RHEA, le modèle DPFT et qui applique la procédure multi-modèle issue de l'algorithme du CERGRENE [8] a été élaboré. Le travail d'exploitation et d'analyse des résultats est actuellement en cours. Les premiers résultats s'avèrent très prometteurs.

Soulignons que ce projet est mené en étroite collaboration avec les spécialistes des services de l'Etat et des laboratoires de recherche français dans les domaines de l'utilisation du radar en hydrologie opérationnelle et de la prévision des crues. Ceux-ci sont invités à participer à toutes les réunions de projet. En outre, dans le cadre de ce projet, une formation à la fois théorique et pratique à l'utilisation de l'Outil est dispensée à une quinzaine d'agents des SAC.

\section{III — SYSTÈMES D'INFORMATION AVANCÉS ET APPROCHES INNOVANTES}

\section{- 3.1 Gestion des pollutions agricoles - projet ISMAP}

Le projet ISMAP (Integrated System for Management of Agricultural Pollution) est un projet EUREKA qui a été conçu en 1991 et qui vient de se terminer (mars 1998). Le projet était bien international (essentiellement franco-italien), le partenariat français a été mené par le LHF pendant la phase de définition et des études puis, pendant la phase de développement de prototype industriel, le relais a été assuré par la Compagnie Générale des Eaux.

La première question importante à laquelle on voulait répondre était : Comment concilier un contexte conflictuel des bénéfices apportés par l'utilisation des engrais et des pesticides avec les risques de contamination des ressources en eau ? La réponse passe par trois volets traités dans le projet :

- un système d'information et d'aide à la décision,

- de nouvelles technologies de détection et de mesure des produits polluants dans l'eau,

- l'optimisation des procédures de traitement des eaux.
La deuxième question, plus importante peut-être, est double : Comment s'adapter à la complexité et aux multiples facettes du problème ? Comment concilier pratiques agricoles, traitement des eaux, réglementation, le principe du développement durable, l'opinion publique ? Il est plus difficile d'y répondre, et la réponse proposée a été amenée par une réflexion hydroinformatique dépassant les limites de la technique ou de la technologie. La réponse " Une approche commune à tous les acteurs industriels, agricoles et publics " demande à être clarifiée pour ne pas être prise pour simple propagande.

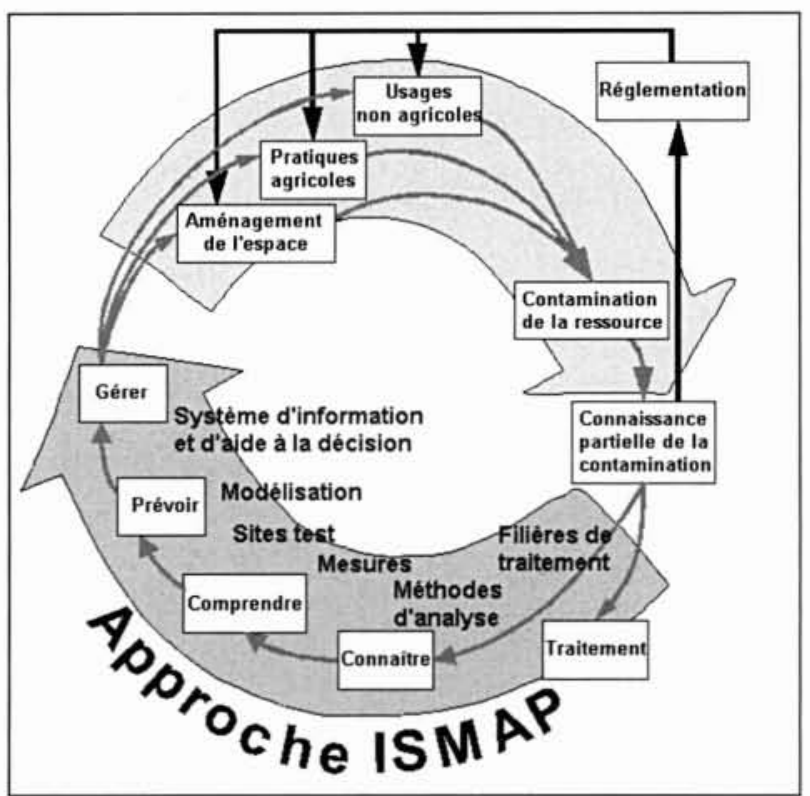

3. Approche ISMAP

Tout d'abord il convient d'énumérer les participants français au projet (sans parler des partenaires italiens) :

Pour les fabricants de produits : Rhône-Poulenc, CIBAGEIGY France, Grande Paroisse ;

Pour les traiteurs d'eau : la CGE :

Pour les réglementations : les Agences de l'Eau ;

Pour les utilisateurs : les associations des agriculteurs, ITCF ; Pour la recherche agricole : CEMAGREF ;

Pour les études : LHF.

Il faut ajouter que les liens ont été maintenus pendant la durée du projet avec CORPEN, FERTIMIEUX et, évidemment, avec les ministères de l'Environnement et de la Recherche.

Le principe permettant de répondre à la question est illustré sur la figure 4 de la "Boucle de Consensus ". Il est fondé sur la notion d'existence d'un " outil objectif ", celui-ci pouvant être une méthodologie, un système de modélisation, un ensemble de procédures. Il faut qu'un tel outil, dans notre cas la méthodologie ISMAP, soit élaboré en commun et accepté par tous les acteurs. Ainsi tous les acteurs admettent que l'outil est impartial et alors on peut chercher des solutions consensuelles en l'employant : chacun est libre de proposer telle ou telle hypothèse (par exemple la quantité des produits à employer par hectare) et la discussion portera sur les conséquences (par exemple le degré de pollution de l'eau à l'exutoire du bassin versant). C'est ce qu'on appelle la " négociation sur le mérite" : on ne met en question ni un outil ni la bonne foi des responsables techniques d'études, etc. Ainsi on dépassionne le débat (9]. 


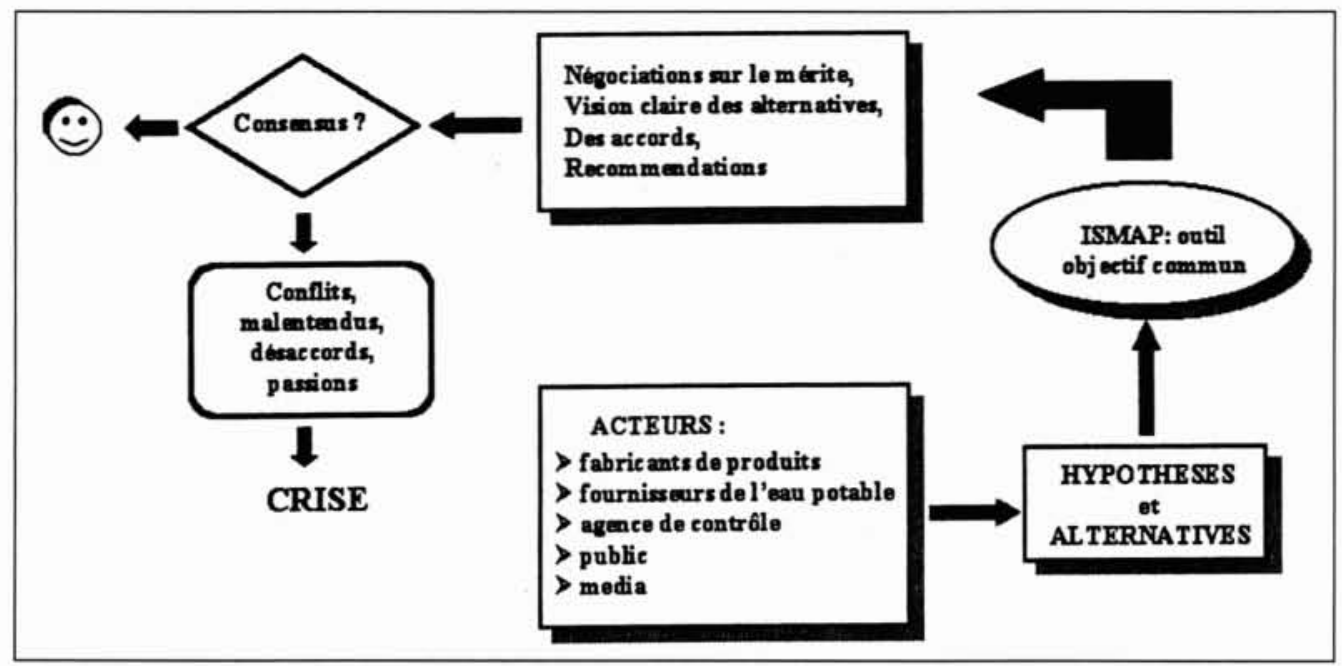

4. Boucle de consensus du Projet ISMAP.

\subsection{Interpréter les résultats de modèles numériques : la logique floue}

Quelle est la valeur, la validité, la certitude, des résultats présentés par les études dans le domaine de ressources en eau ? Et notamment des résultats de modèles numériques ? Quand un prestataire de services fournit à son client un hydrogramme de crue, ou l'enveloppe des élévations maximales le long d'un cours d'eau obtenues par un modèle, quelle est l'exactitude de ce résultat? Nous savons tous que de tels résultats sont forcément imprécis et que l'imprécision est fonction de nombreux facteurs. Nous savons aussi qu'il n'est pas toujours " politiquement correct " de présenter un résultat encadré par un intervalle d'imprécision, sous prétexte qu'une telle présentation sème le doute quant à la validité de résultat. Or, il serait souhaitable que nous soyons dans une situation inverse : un résultat " unique " et " sûr " de la simulation d'une crue doit être suspect par définition à toute personne qui réfléchit sérieusement sur le sujet, même si cette personne n'est pas un ingénieur. Un travail important qui nous attend tous : introduire la culture de compréhension et de gestion des imprécisions, voire des incertitudes dans les résultats de la modélisation. Le premier pas dans ce sens consiste en l'élaboration d'une méthodologie permettant d'évaluer d'une manière fiable, applicable et fondée scientifiquement les imprécisions sur les résultats de modélisation. Les débuts sont faits dans ce sens dans notre domaine avec le travail effectué au LHF sur l'application de la logique et de l'algèbre de sous-ensembles flous $[10,11]$. Cette méthode permet de quantifier les imprécisions sur les résultats issus des modèles mathématiques et d'évaluer la confiance que l'on peut leur accorder. Les premières applications à l'estimation de la vulnérabilité de la ressource en eau face à des pollutions diffuses d'origine agricole ont démontré la généralité de cette méthodologie et il est fort probable sinon certain que celle-ci trouvera sa place dans le corpus de l'Ecole Française de l'Eau dans l'avenir comme une approche systématique, en contribuant ainsi à la création de la culture de gestion de l'incertain dont on a parlé plus haut.

\section{IV — NOUVELLE APPROCHE FRANÇAISE DANS LES ÉTUDES DES RIVIÈRES, RETOMBÉES INTERNATIONALES}

En cinq années, l'approche française des études fluviales a considérablement changée, elle a même été bouleversée grâce à l'apparition sur le marché des logiciels et des études du système de modélisation TELEMAC $2 \mathrm{D}$ développé par le LNH/EDF de Chatou, et aussi grâce au travail considérable du LHF pour promouvoir non seulement les ventes de licences mais aussi les applications fluviales. La modélisation numérique des rivières était réservée, jusqu'à récemment, à l'emploi des modèles numériques unidimensionnels ou, éventuellement, multidirectionnels à casiers. On peut nommer, dans cette catégorie : CARIMA et HYDRA (comme représentants des développements français), ISIS (GB), SOBEK (NL), MIKEII (DK). Les limitations de ces modèles sont évidentes : ils ne permettent pas de reproduire en détail des phénomènes à caractère bidimensionnel et pour cette raison leurs résultats ne peuvent pas être utilisés à des fins d'expertise sédimentologique et morphologique à l'échelle locale, seule l'évolution de profil en long sur des dizaines de kilomètres pouvant être considérée. Ainsi, dès qu'il s'agissait de problèmes locaux (concernant les longueurs de quelques kilomètres), seule la modélisation physique, généralement trop onéreuse, pouvait être envisagée pour les études de fonds mobiles. Par ailleurs les modèles multidirectionnels à casiers peuvent induire leur utilisateur en erreur car non seulement ils ne tiennent pas compte des termes d'inertie dans leur partie " bidimensionnelle " mais déforment aussi les sections de passage entre les casiers. Ainsi les champs de vitesses " quasi bidimensionnelles " qu'ils fournissent sont très éloignés de la réalité. La même remarque vaut pour les élévations de la surface libre (les " casiers" de ces modèles ont la surface libre, par définition, horizontale). TELEMAC 2D est un logiciel par définition bidimensionnel et à l'origine il était développé pour être employé dans les zones côtières. Bâti sur la méthode des éléments finis, il est particulièrement efficace pour les zones à géométrie complexe, ce qui est généralement le cas de rivières quand on considère celles-ci à l'échelle de deux dimensions horizontales. On peut parler ici d'une nouvelle génération des modèles bidimensionnels venant après CYTHERE ES1 (développé dans les années 1980-85 en commun par le LNH/EDF et SOGREAH, fondé sur le principe des différences finies, aujourd'hui abandonné) et MIKE21 (développé depuis 1975 par le DHI, à la base des différences finies, largement employé pour les zones côtières). Le fait nouveau est d'avoir prouvé par LHF l'applicabilité parfaite de TELEMAC 2D à des études fluviales 


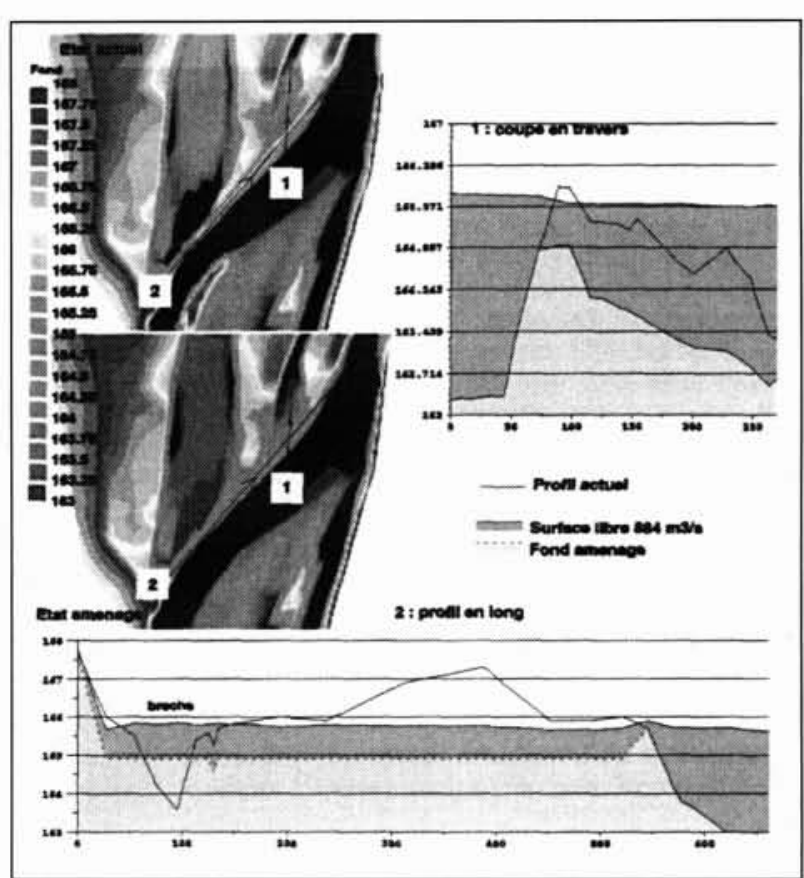

5. Aménagement de la chevrette de Givry (site amont).

concernant la morpho-sédimentologie, les détails de la dynamique des champs d'inondation, des traversées des vallées inondables par les remblais routiers ou par ceux des chemins de fer. De plus on a pu démontrer que le ratio coûts/bénéfices d'études effectuées en l'employant est acceptable pour le marché concurrentiel français dans le domaine fluvial. On peut citer ici les exemples d'études effectuées par le LHF en utilisant TELEMAC 2D: la traversée de la zone de confluence de I'lle et de la Dordogne par l'autoroute A89, les études des chevrettes à Givry (Fig. 5) et Ousson sur la Loire, la traversée d'Orléans, l'étude des écoulements au voisinage du Pont de Ners sur Gardon, l'étude de scénarios d'aménagement de la Durance.

La percée d'une nouvelle approche d'ingénierie fluviale est confirmée par d'autres études en France (l'Orne par SOGREAH, de la Rance par le LNH), ainsi que par le nombre croissant des licenciés du logiciel parmi les Bureaux d'Etudes français (SOGREAH, BCEOM) ou implantations de recherche à l'étranger (Ecole Polytechnique de Varsovie, Franzius Institute, Université de Hanovre en Allemagne, ENEL/CRIS en Italie, Instituto Superior Tecnico au Portugal) [12].

L'exemple des applications de TELEMAC en Pologne est particulièrement représentatif de cette nouvelle percée. Le réservoir Zegrzynski est une retenue artificielle d'une longueur de $30 \mathrm{~km}$ et possédant le volume total de 94 millions de $\mathrm{m}^{3}$ créée sur deux des plus grandes rivières de cette région : Bug and Narew (Fig. 6). Situé dans la proximité de Varsovie il représente une source stratégique de l'alimentation de la capitale polonaise ( 2 millions d'habitants) en eau potable et un endroit très important pour les sports aquatiques et la détente. Pour cette raison, l'Institut des Systèmes du Génie de l'Environnement de l'Ecole Polytechnique de Varsovie est impliqué dans l'établissement d'un modèle numérique de la qualité de l'eau du réservoir orienté vers une meilleure représentation des processus de l'eutrophisation.

Le modèle d'écosystème de la retenue est étudié à l'aide de TELEMAC 2D. Dans une première étape un modèle

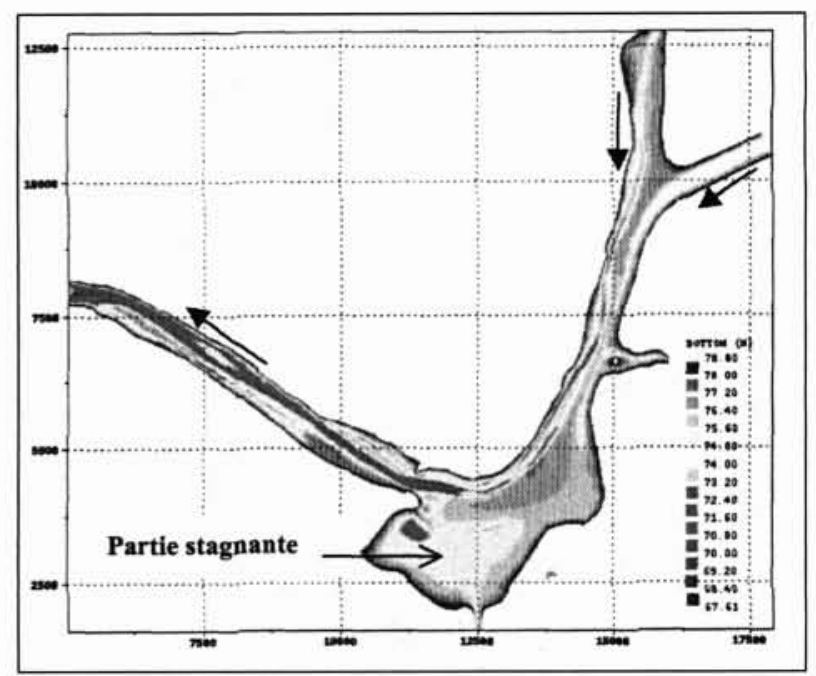

6. Situation bathymétrique de la retenue Zegrzynski.

hydrodynamique du réservoir à été construit sur la base de résultats d'une campagne de mesures bathymétriques actuellement est mené un travail de couplage du modèle hydrodynamique avec le module de qualité SUBIEF-2D du système TELEMAC.

\section{V — COMPLÉMENTARITÉ DES SYSTÈMES HYDROLOGIQUES ET HYDRAULIQUES}

Un exemple de cette approche appliquée récemment par le LHF sont des études hydrologiques et hydrodynamiques et prédictions de l'augmentation du niveau de la Mer Caspienne. En effet, depuis 1978 on observe une augmentation systématique du niveau de la Mer Caspienne et une aggravation de la situation en ce qui concerne l'inondation des zones habitées. Les experts ne sont pas d'accord sur les origines et l'évolution future de ce phénomène.

Dans le cadre du programme INCO COPERNICUS de coopération des pays membres de l'Union Européenne avec les pays de l'Europe centrale et avec des Nouveaux Etats Indépendants, LHF a conçu un projet multidisciplinaire de recherche qui a pour but une meilleure connaissance des facteurs hydro-météorologiques et hydrodynamiques impliqués dans ce phénomène.

Le Projet [13] mené en collaboration entre LHF et I'Université de Bochum (Allemagne), Institut d'Hydrologie de Wallingford (Grande Bretagne), I'Institut Russe d'Hydrologie de Saint Petersburg (Fédération Russe) et le Service Hydro-Météorologique de Turkmenistan tente :

- d'établir un bilan détaillé de la Mer Caspienne ;

- d'étudier les échanges à l'intérieur du bassin de la Mer ; - d'évaluer, grâce à l'application de techniques de la télédétection, l'impact éventuel des activités humaines dans le bassin de la Volga (apport annuel de $80 \%$ du volume total entrant dans la Mer Caspienne) ;

- de déterminer les scénarios d'évolution du niveau de la Mer en tenant compte des scénarios des changements climatiques futurs.

Pour atteindre ces objectifs, LHF a proposer d'utiliser d'une manière conjointe des modèles hydrologiques du type statistique avec la modélisation bi- et tridimensionnelle de la Mer à I'aide de TELEMAC 2D/3D [14]. 


\section{VI $\square$ CONCLUSION}

La globalisation d'informations et de transferts des connaissances font que les acteurs nationaux participant à l'évolution de l'Ecole française de l'eau ne peuvent que rarement se limiter au territoire national et que les transferts se font dans les deux sens avec l'étranger. L'imagination appuyée sur la tradition française d'innovation, pour peu qu'elle soit relayée par une ouverture vers d'autres technologies et surtout les technologies de pointe, permet aux " petits" d'avoir une influence et un rayonnement tout à fait respectables ! Cette globalisation d'information fait que les applications des technologies de pointe dans les pays jusqu'à récemment catégorisés “ de tiers monde " sont indispensables pour y maintenir la crédibilité des applications plus classiques venant de notre pays.

\section{Références bibliographiques}

[1] European Commission, DGXIII (December 1997), Project Catalogue, Telematics Applications for the Environment, Telematics Applications Programme 1994-1998, DGXIII-C6, Transport \& Environment Telematics, Bruxelles.

[2] RAHUEL, J.L. CHAILLET, P. et ERLICH M., "Modélisation du système hydrographique du delta du Yangtze pour la prévision opérationnelle des inondations de Shanghấ", 24ème Journées de l'Hydraulique, "L'Eau, I'Homme et la Nature". Thème 2, Les chemins de I'eau au titre de la question III : "Deltas, estuaires et littoral", 18-20 septembre 1996, Paris, SHF.

[3] RAHUEL, J.L., CHAILLET, P., WU, Chaojun (1996) "An Integrated Real-time Data Acquisition and Forecast System for Flood Prevention in Shanghai", Proceedings of Southeast Asia Regional Workshop on Urban Hydrology, 9-12 juillet 1996, Shanghaï, Chine, Hohai University Press, pp. 17-31.

[4] CUNGE, J., NEGRE, J.L, DUBAND, D. et KAPFER, A. (1994) "Une Méthodologie et un Système Intégré de Prévision de Crues-Eclairs pour les Services d'Annonces des Crues", Crues et Inondations, SHF, pp. 607-610, Congrès SHF, Nimes, 14-16 Septembre 1994.
[5] RODRIGUEZ, J.Y., OBLED, Ch. et SEMPERE-TORRES, D. (1991) "Prévision des crues dans les petits bassins versants de montagne : prise en compte de la variabilité spatiale des pluies et des mécanismes de production par l'approche DPFT", Houille Blanche, $\mathrm{N}^{\circ} 5,341-348$, SHF.

[6] CUNGE, J., ERLICH, M., RAHUEL, J.L. (1994) "Towards Scenariobased Real-time Forecasting for Flood-affected Basins using a Multimodel Integrated System", 2nd International Conference on River Flood Hydraulics, Chapitre 2 "Flood Forecasting". pp. 77-91, York, England.

[7] RHEA, 1994, "Le logiciel CALAMAR I et ses fonctions", Documentation du stage " Mesure et Prévision des crues ", Ecole Centrale de Lyon et Ecole des Mines de St.Etienne.

[8] ROCHE, P.A. et TAMIN, R. (1986), "Procédures de décision multimodèles applicables à la prévision des crues en temps-réel", Rapport d'étude, CERGRENE, ENPC.

[9] BOISSON, M. et VANDEVELDE, T. (1996) "Le système ISMAP : un système intégré d'aide à la gestion des pollutions diffuses d'origine agricole". ESRA'96, L'eau souterraine en région Agricole, Poitiers, 9-12 septembre 1996.

[10] FREISSINET, C. (1997) "Estimation des imprécisions dans la modélisation du devenir des produits phytosanitaires dans les sols : une méthode fondée sur la logique floue". Thèse de doctorat de l'Université Joseph Fourier, Grenoble.

[11] FREISSINET, C., ERLICH M. et VAUCLIN M.(1997), "Application of a fuzzy set approach to the evaluation of imprecisions in pesticide groundwater pollution using a screening model", in Proceedings of European Symposium on Intelligent Techniques, March 20-21, 1997, Bari, pp. 103-107. ERUDIT, Aachen.

[12] DAVID, E., LANG, P. et SAUVAGET, P. (1997) "New trends in the integrated management of inland waters: the possibilities of 2D F.E. flow modelling", International Conference on Management of Transboundary Waters in Europe, Mrzezyno, Pologne, 22-25 septembre 1997.

[13] "Hydrological and hydrodynamic studies and predictions of the Caspian sea water level rise - impact of climate factor and man's activities (CASSEAS)". INCO COPERNICUS Projects Catalogue, vol. II, EC DG XII, 1997.

[14] SAUVAGET, P., DAVID, E. et LANG, P.(1996) "Etude numérique du fonctionnement hydrodynamique d'un plan d'eau côtier profond méditerranéen : la baie de Monaco". 24èmes Journées de I'Hydraulique " L'Eau, l'Homme et la Nature ", colloque de la SHF, Paris. 